\title{
Identification of genes associated with the effect of inflammation on the neurotransmission of vascular smooth muscle cell
}

\author{
SHUJIE GAN ${ }^{1}$, SHENLONG QIU $^{1}$, YIWEN FENG ${ }^{1}$, YANPING ZHANG $^{1}$, \\ QIN QIAN $^{1}$, ZHONG WAN $^{1}$ and JINGDONG TANG ${ }^{2}$ \\ ${ }^{1}$ Department of Vascular Surgery, The First People's Hospital, Shanghai Jiao Tong University, Shanghai 200080; \\ ${ }^{2}$ Department of Vascular Surgery, Shanghai Pudong Hospital, Fudan University, \\ Pudong Medical Center, Shanghai 201399, P.R. China
}

Received August 11, 2015; Accepted December 9, 2016

DOI: $10.3892 /$ etm.2017.4138

\begin{abstract}
Vascular smooth muscle cell (VSMC) accumulation and hypertrophy are common in vascular disorders, and inflammation has a crucial role in the development of these diseases. To investigate the effect of inflammation on the neurotransmission of VSMC, bioinformatic analysis was performed, following next generation sequencing. Genes of lipopolysaccharide (LPS)-treated A7r5 cells and phosphate-buffered saline (PBS)-treated A7r5 cells were sequenced via next generation sequencing, and each assay was repeated three times. Differentially expressed genes (DEGs) were obtained using the NOISeq package in R. Subsequently, their potential functions were predicted by functional and pathway enrichment analyses using the Database for Annotation, Visualization and Integrated Discovery online tool. Interaction relationships of the proteins enriched in pathways associated with neurological diseases, the proteins which had interaction relationships with adrenoceptor $\alpha$ 1D (ADRA1D) or calcium voltage-gated channel subunit $\alpha 1 \mathrm{~S}(C A C N A 1 S)$, separately, were obtained from STRING, and protein-protein interaction (PPI) networks were constructed using Cytoscape software. A total of 2,038 DEGs, including 1,094 upregulated and 944 downregulated genes in the LPS treatment group were identified when compared with the control group. Enrichment analyses showed that NADH:Ubiquinone Oxidoreductase Core Subunit V2 (NDUFV2) was involved in several neurological diseases, including oxidative phosphorylation, Alzheimer's disease, Parkinson's disease and Huntington's disease. Furthermore, NDUFV2 (degree, 20) had a higher degree in the PPI network
\end{abstract}

Correspondence to: Dr Jingdong Tang, Department of Vascular Surgery, Shanghai Pudong Hospital, Fudan University, Pudong Medical Center, 2800 Gongwei Road, Pudong New Area, Shanghai 201399, P.R. China

E-mail: drtangjingdong@126.com

Key words: vascular smooth muscle cell, inflammation, next generation sequencing, differentially expressed genes, protein-protein interaction network for DEGs enriched in pathways associated with neurological diseases. In the PPI network for ADRA1D, CACNAIS and the DEGs interacting with them, prohibitin $(P H B)$, oxytocin receptor $(O X T R)$, collapsin response mediator protein 1 (CRMP1) and dihydropyrimidinase like 2 (DPYSL2) had interaction relationships with both $A D R A 1 D$ and CACNAIS. To conclude, the present study revealed that $N D U F V 2, P H B$, OXTR, CRMP1 and DPYSL2 may have key roles in the effect of inflammation on neurotransmission of VSMC.

\section{Introduction}

As a highly specialized cell in mature animals, the vascular smooth muscle cell (VSMC) has a principal function of contraction; however, production of matrix components of the blood vessel wall and proliferation becomes the primary function of VSMCs during vasculogenesis (1). Abnormal contraction of SMC is a major incentive of vasospasm of the cerebral and coronary arteries, as well as hypertension (2). VSMC accumulation and hypertrophy are common in vascular disorders, such as atherosclerosis, hypertension, restenosis $(3,4)$ and inflammation, which can be induced by hypoxia and has crucial roles in the development of these diseases $(5,6)$. Thus, there is an urgency to elucidate the effect of inflammation on the neurotransmission of VSMCs.

Several pharmacological agents are capable of inducing inflammation. In human aortic smooth muscle cells, lipopolysaccharide (LPS) promotes the production of nitric oxide (NO) and Toll-like receptor 4 (TLR4) expression, inducing inflammatory responses (4). A previous study demonstrated that propranolol has a negative chronotropic effect on the expression levels of pro-inflammatory cytokines after myocardial infarction (MI) in rats (7). In rat aorta, $\beta$-adrenoceptors were overstimulated by the agonist, isoproterenol, which resulted in an increase of vascular inflammatory mediators, such as interleukin (IL)-1 $\beta$, IL-6 and nuclear factor $\kappa \mathrm{B}(\mathrm{NF}-\kappa \mathrm{B})(8)$. At a concentration of $20 \mu \mathrm{g} / \mathrm{ml}$, the non-selective $\beta$-adrenergic receptor agonist, propranolol, was revealed to suppress cell growth of infantile hemangioma endothelial cells (IHECs) in vitro once the proliferation stage of IHECs has been affected for between 72 and $96 \mathrm{~h}$, whereas isoproterenol yielded the opposite results (9). 
Previous reports have been conducted to survey the effect of inflammation on VSMC. As an adipocytokine, extracellular pre-B cell colony-enhancing factor/nicotinamide phosphoribosyltransferase/visfatin (ePBEF/NAMPT/visfatin) functions as a direct contributor to vascular inflammation via its NAMPT activity (10). Through regulating vascular cell activation and inflammatory cell recruitment, the adhesion protein, cluster differentiation (CD) 44 , has an important role in the development of atherosclerotic diseases (11). By inhibiting the activation of hypoxia-inducible factor- $1 \alpha$, transcription factors, NF- $\mathrm{kB}$ and activator protein-1 (AP-1), and 3-hydroxy-3-methylglutaryl-coenzyme A (HMG-CoA) reductase inhibitors have anti-proliferative and anti-inflammatory effects on human endothelial and vascular smooth muscle cells; thus, statins can be used to treat atherosclerosis (12).

In the present study, next generation sequencing was conducted to obtain sequence data. Differentially expressed genes (DEGs) between the LPS treatment group and the control group were screened and their functions were predicted by enrichment analyses. Moreover, a protein-protein interaction (PPI) network was constructed to investigate the interaction relationships between these DEGs.

\section{Materials and methods}

Cell cultivation. Rat VSMC cell line A7r5, which was purchased from Shanghai enzyme research Biotechnology Co., Ltd. (Shanghai, China), was cultivated in Dulbecco's modified Eagle medium (DMEM) (Gibco; Thermo Fisher Scientific, Inc., Waltham, MA, USA) and maintained at $37^{\circ} \mathrm{C}$ using an incubator (Thermo Fisher Scientific, Inc). DMEM was discarded, and A7r5 cells were digested with pancreatin (Gibco; Thermo Fisher Scientific, Inc) for $5 \mathrm{~min}$. Subsequently, 6-fold DMEM was added to terminate digestion and cells were centrifuged at room temperature with $157 \times g$ for $5 \mathrm{~min}$, and the supernatant and resuspension was discarded. A7r 5 cells were cultured in new culture flasks in a $5 \% \mathrm{CO}_{2}$ incubator at $37^{\circ} \mathrm{C}$ (Thermo Fisher Scientific, Inc.). Using the frozen stock solution made of $10 \%$ dimethyl sulfoxide (DMSO; Sigma-Aldrich; Merck Millipore, Darmstadt, Germany) and $90 \%$ fetal bovine serum (FBS) (Gibco; Thermo Fisher Scientific, Inc.), A7r5 cells were resuspended at a density of $5 \times 10^{6}$ cells $/ \mathrm{ml}$. Following stewing at $4^{\circ} \mathrm{C}$ for $10 \mathrm{~min}$, cells were cryopreserved at $-20^{\circ} \mathrm{C}$ for $2 \mathrm{~h}$ and stored at $-80^{\circ} \mathrm{C}$ overnight.

Calcium detection. A7r5 cells were inoculated into confocal plates (Wohong Biotechnology Co., Ltd., Shanghai, China) $\left(2 \times 10^{4}\right.$ cells/plate) and cultivated overnight. Cells were washed with phosphate-buffered saline (PBS) twice with $100 \mu$ l PBS containing $5 \mu \mathrm{mol} / 1$ Fluo-4/AM and lucifugally incubated at $37^{\circ} \mathrm{C}$ in a $5 \% \mathrm{CO}_{2}$ incubator for $45 \mathrm{~min}$. Subsequently, cells were washed with PBS twice again and induced by LPS $(100 \mu \mathrm{g} / \mathrm{ml})$ for $30 \mathrm{~min}$. In the control groups, PBS was used instead of LPS. Under confocal laser scanning microscope, A7r 5 cells were observed and images were captured before and after treatment with isoprenaline $(10 \mu \mathrm{mol} / 1$, Melone pharmaceutical, Co., Ltd., Dalian, China) or propranolol (10 $\mu \mathrm{mol} / 1$, Melone pharmaceutical, Co., Ltd.).
Cell hypoxia treatment. Cells were inoculated with $0.5 \%$ FBS in confocal plates $\left(4 \times 10^{5}\right.$ cells/plate). After being cultivated for $24 \mathrm{~h}$, the $0.5 \%$ FBS medium was replaced with $10 \%$ FBS and cells were treated with cobalt dichloride $(200 \mu \mathrm{mol} / \mathrm{l})$ for $24 \mathrm{~h}$. Subsequently, cells were induced by LPS $(100 \mu \mathrm{g} / \mathrm{ml})$ for $30 \mathrm{~min}$; however, cells in the control group received PBS. After cell digestion by pancreatin (Gibco; Thermo Fisher Scientific, Inc.) for $5 \mathrm{~min}$, the mixture was centrifuged at room temperature at $157 \mathrm{x} g$ for $5 \mathrm{~min}$. Then, cells were washed three times with DMEM at room temperature and further centrifuged at room temperature at $157 \mathrm{x} g$ for $5 \mathrm{~min}$. The supernatant was discarded and A7r5 cells were preserved in TRIzol (Invitrogen; Thermo fisher Scientific, Inc.) at $-80^{\circ} \mathrm{C}$. Each assay was performed in triplicate.

RNA isolation and RNA-sequence library construction. Total RNA of the LPS treatment group and the control group were extracted using a TRIzol total RNA extraction kit (Invitrogen; Thermo Fisher Scientific, Inc.) according to the manufacturer's instructions. The integrity and purity of total RNA was detected using $2 \%$ agarose gel electrophoresis and a spectrophotometer (Merinton Instrument, Ltd., Beijing, China), respectively. The RNA-sequence library was constructed using methods described in a previous study (13). Subsequently, DNA cluster amplification was performed and high-throughput sequencing was conducted for the library, using Illumina Hiseq 2000 100PE (Illumina Inc., San Diego, CA, USA). After the raw data was obtained, sequences containing an adaptor, $>50 \%$ low quality bases and $>3 \%$ unknown bases were filtered out.

DEGs screening. Following filtering, the sequences were mapped to the rat genome (rn5), using bowtiel in TopHat software (version 2.1.0, accessible at http://ccb.jhu.edu/software/tophat/index.shtml) (14). The maximum read mismatch number was set at 2 , and the parameter 'max-multihits' was set at 1 (15). And the other parameters were set to defaults. Combining with annotation information of rn5 in ensemble, expression of each sample was annotated using Cufflinks software (version 2.2.1, accessible at http://cole-trapnell-lab.github.io/cufflinks/) (16) under the default parameter values. The NOISeq package (version 2.18.0, accessible at http://www.bioconductor. org/packages/release/bioc/html/NOISeq.html) (17) in R was used to screen the DEGs between the LPS treatment group and the control group. The probability of a gene being DEGs (q) was set to 0.99 .

Functional and pathway enrichment analysis. Gene Ontology (GO), which consists of three categories, including biological process (BP), molecular function (MF) and cellular component (CC), is used to generate the vocabulary that can be applied to all eukaryotes (18). As a database, Kyoto Encyclopedia of Genes and Genomes (KEGG) includes information of known genes and their biochemical functionalities (19). Using the Database for Annotation, Visualization, and Integrated Discovery (DAVID) online tool (20), GO and KEGG pathway enrichment analyses were conducted for the upregulated and downregulated genes between the LPS treatment and control groups, respectively. $\mathrm{P}<0.01$ was used as the cut-off criterion. 


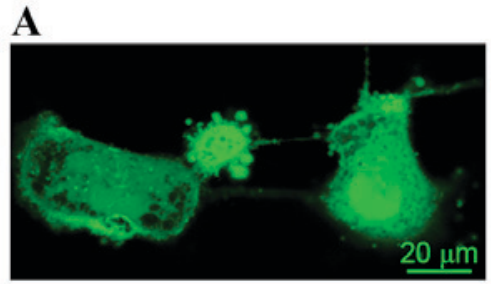

D

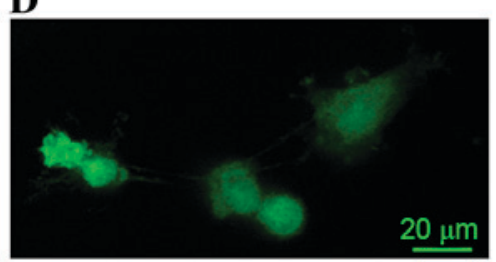

B

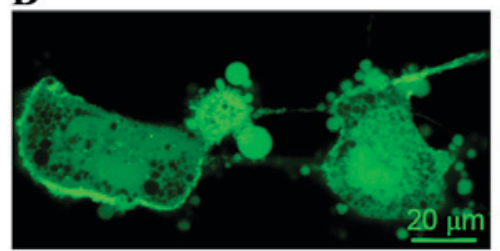

E

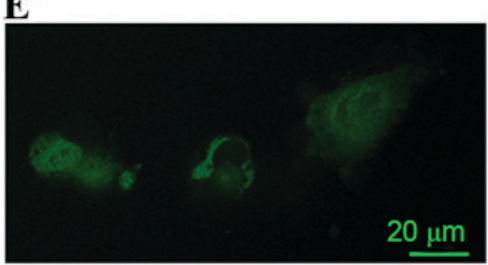

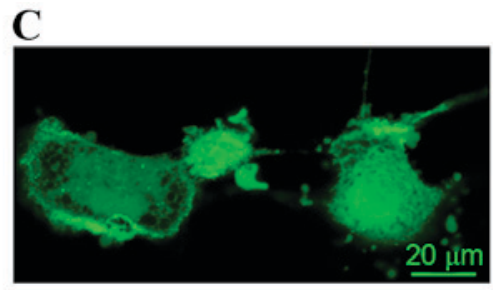

F

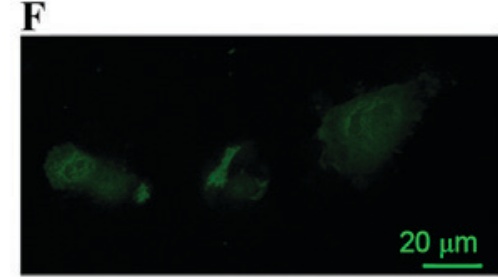

Figure 1. Calcium signals in A7r5 cells observed under confocal laser scanning microscope. (A) Calcium signals in A7r5 cells in the PBS group before treatment with isoprenaline $(10 \mathrm{~mol} / \mathrm{l})$ or propranolol $(10 \mathrm{~mol} / \mathrm{l})$. (B) Calcium signals in A7r5 cells of the PBS group after isoprenaline treatment. (C) Calcium signals in A7r5 cells in the PBS group after being treated with propranolol. (D) Calcium signals in A7r5 cells in the LPS group before being treated with isoprenaline (10 mol/l) or propranolol (10 mol/l). (E) Calcium signals in A7r5 cells in the LPS group after isoprenaline treatment. (F) Calcium signals in A7r5 cells in the LPS group after propranolol treatment. PBS, phosphate-buffered saline; LPS, lipopolysaccharide.

PPI network construction. Using STRING online software (version 10.0, accessible at http://www.string-db.org/) (21), interaction relationships of the proteins encoded by the DEGs were searched, and the required confidence (combined score) $>0.1$ was used as the cut-off criterion. Subsequently, PPI network was visualized using Cytoscape (version 3.2.0, accessible at http://www.cytoscape.org/) (22). Proteins in the network were named as nodes and the degree of a node was equal to the number of nodes interacted with it. Moreover, the nodes with degrees higher than 20 were defined as hub nodes.

\section{Results}

Calcium detection. In the PBS control group, A7r5 cells prior to treatment with isoprenaline or propranolol are indicated in Fig. 1A. In A7r5 cells treated with isoprenaline, the concentration of calcium decreased (Fig. 1B); however, the level of calcium in propranolol-treated A7r5 cells increased (Fig. 1C).

The LPS-treated group (Fig. 1D-F) exhibited a reduced calcium signal prior to treatment with isoprenaline or propranolol when compared with the PBS control group (Fig. 1D). In the A7r5 cells that were treated with isoprenaline, the concentration of calcium decreased (Fig. 1E). However, the level of calcium in the A7r5 cells treated with propranolol had no change when compared with the LPS group cells treated with isoprenaline (Fig. 1F).

DEGs analysis. Compared with the control PBS group, a total of 2,038 DEGs, including 1,094 upregulated and 944 downregulated genes were identified in the LPS-treated group. These findings indicated that the number of upregulated genes was markedly higher in comparison to the number of downregulated genes.

Functional and pathway enrichment analysis. The enriched GO functions for upregulated genes are listed in Table I. The enriched functions in the BP category included chemical
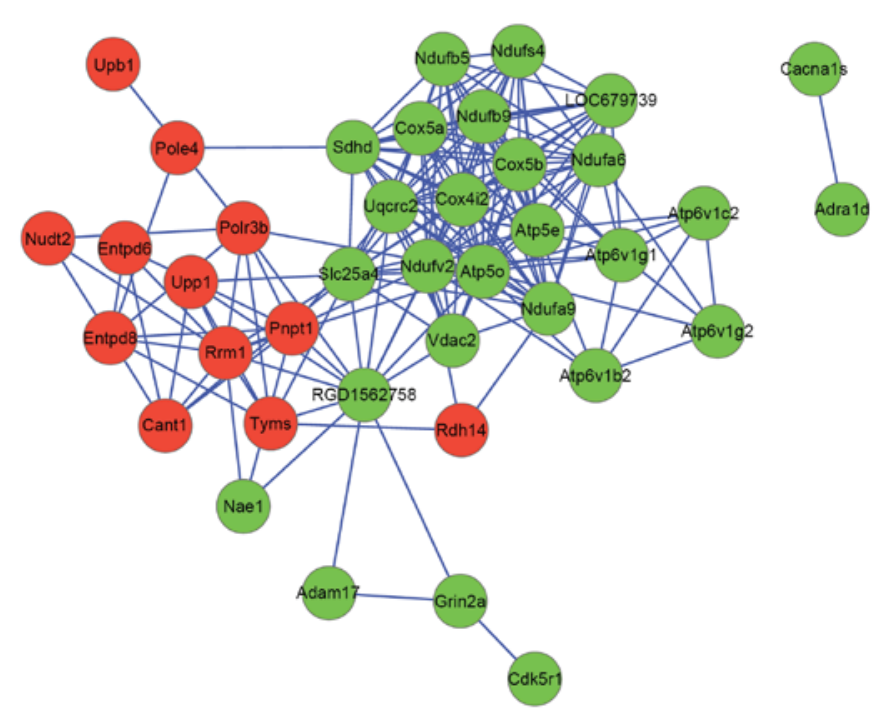

Figure 2. Protein-protein interaction network for differentially expressed genes enriched in pathways associated with neurological diseases. Red circles and green circles represent up-regulated and down-regulated genes, respectively.

homeostasis $(\mathrm{P}=4.81 \mathrm{E}-04)$, mitogen-activated protein kinase kinase kinase cascade $(\mathrm{P}=7.85 \mathrm{E}-04)$ and nucleoside monophosphate metabolic process $(\mathrm{P}=8.39 \mathrm{E}-04)$. The enriched functions in the $\mathrm{CC}$ category included condensed chromosome kinetochore $(\mathrm{P}=1.33 \mathrm{E}-04)$, chromosome, centromeric region $(\mathrm{P}=1.63 \mathrm{E}-04)$ and plasma membrane $(\mathrm{P}=2.96 \mathrm{E}-04)$. Enriched functions in the MF category included hydrolase activity, acting on carbon-nitrogen (but not peptide) bonds in linear amidines $(\mathrm{P}=1.40 \mathrm{E}-04)$, quaternary ammonium group binding $(\mathrm{P}=8.28 \mathrm{E}-04)$ and phosphatidylcholine binding $(\mathrm{P}=9.81 \mathrm{E}-04)$.

The enriched KEGG pathways for upregulated genes are also listed in Table I, including purine metabolism $(\mathrm{P}=3.13 \mathrm{E}-04)$, neuroactive ligand-receptor interaction $(\mathrm{P}=0.003996)$ and pyrimidine metabolism $(\mathrm{P}=0.007245)$. 


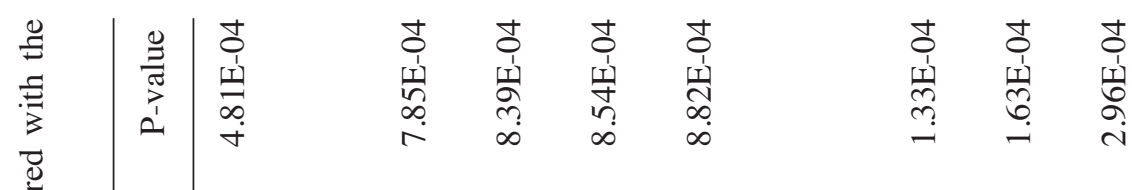

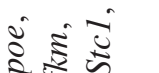

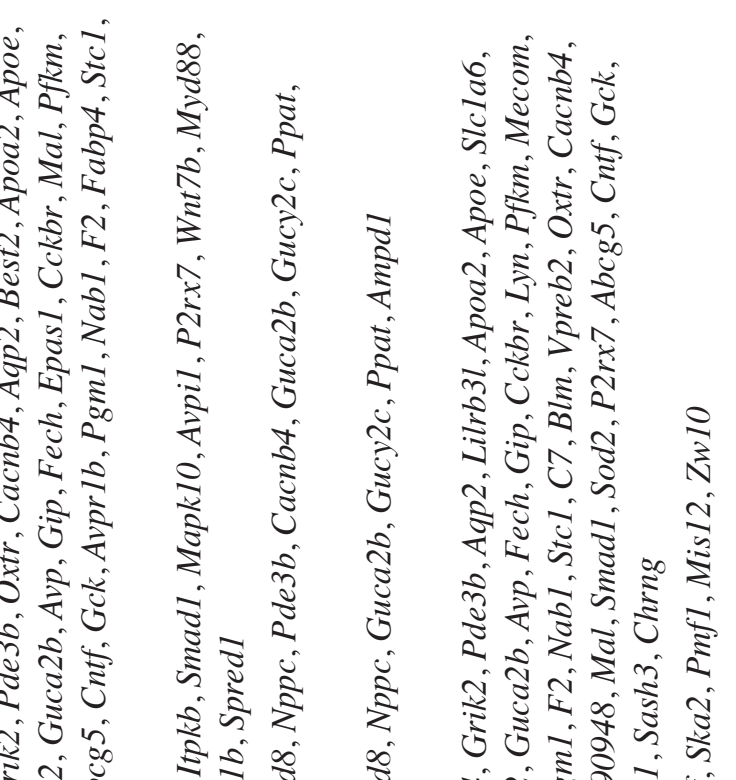

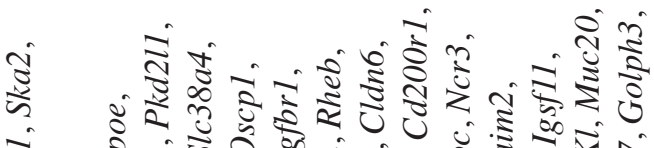

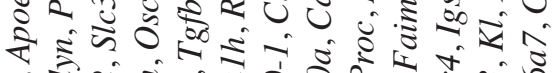

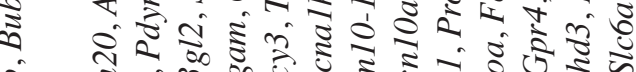

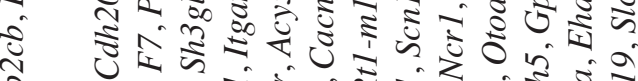
₹ o.

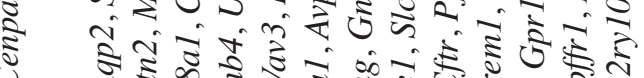

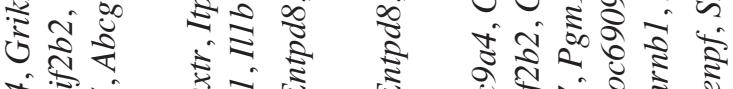

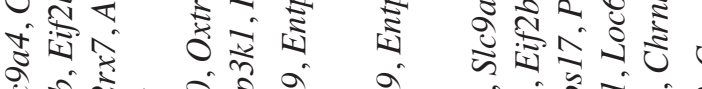

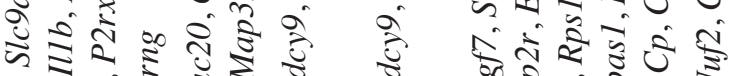

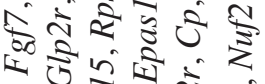

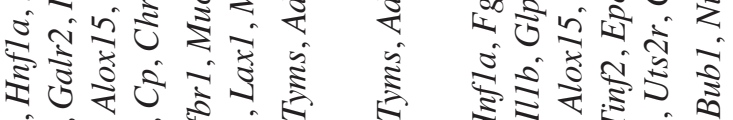

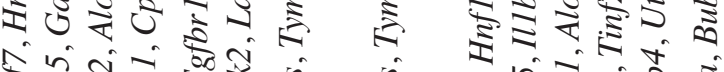

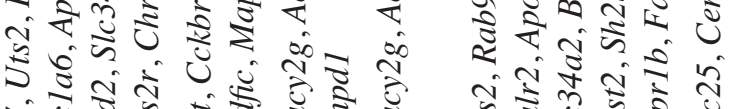

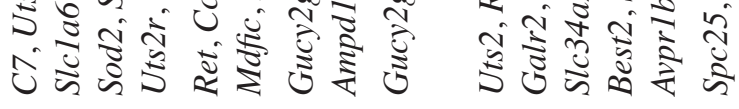

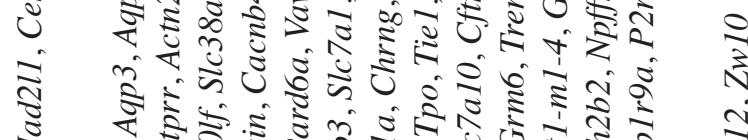

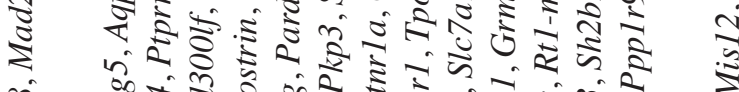

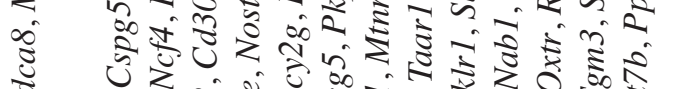

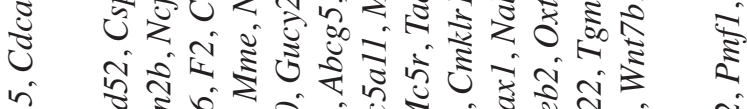

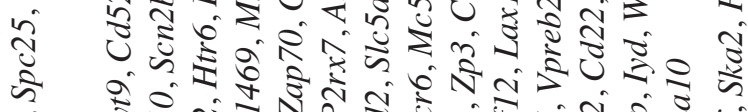

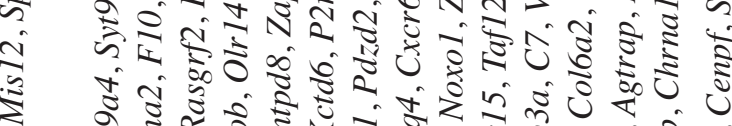

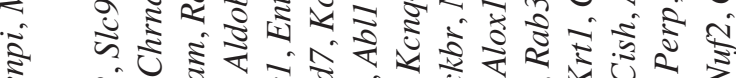

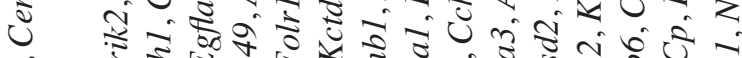

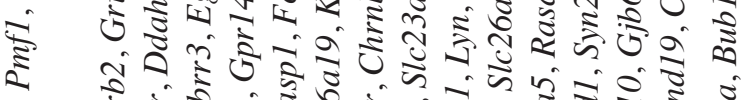

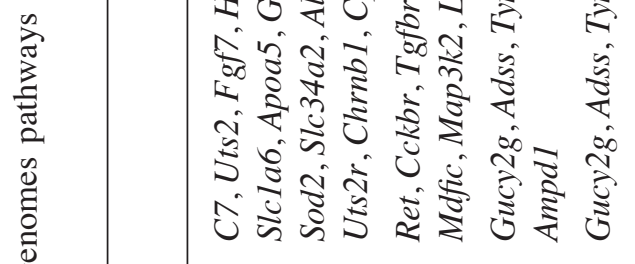
5 כ 过

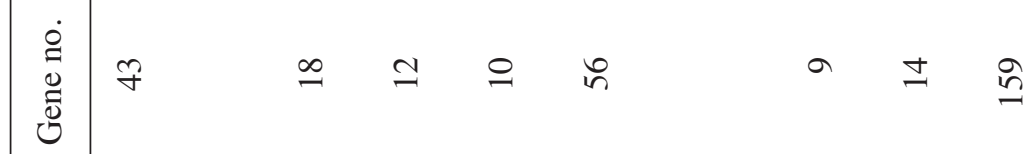
4

:
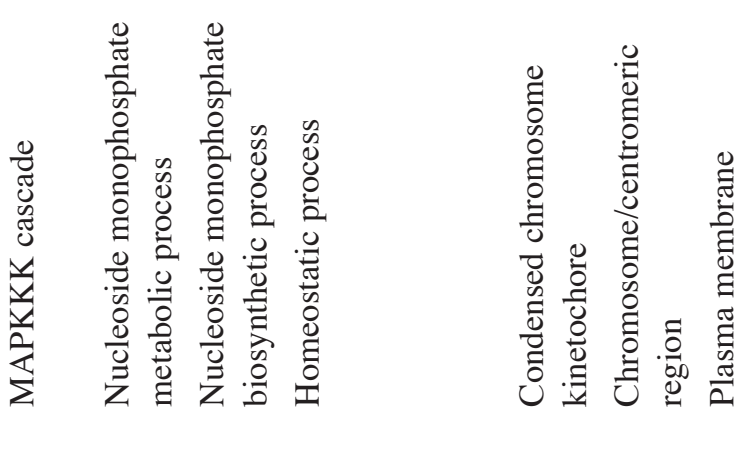
The enriched GO functions for downregulated genes are presented Table II. The enriched functions in the BP category included oxidation reduction $(\mathrm{P}=1.19 \mathrm{E}-05)$, cofactor metabolic process $(\mathrm{P}=0.001298)$ and erythrocyte homeostasis $(\mathrm{P}=0.0013)$. Enriched functions in the CC category included mitochondrion $(\mathrm{P}=6.87 \mathrm{E}-07)$, organelle membrane $(\mathrm{P}=6.03 \mathrm{E}-06)$ and mitochondrial part $(\mathrm{P}=1.67 \mathrm{E}-05)$. The enriched functions in the MF category included nicotinamide adenine dinucleotide or nicotinamide adenine dinucleotide hydride binding $(\mathrm{P}=3.63 \mathrm{E}-04)$, protein homodimerization activity $(\mathrm{P}=4.08 \mathrm{E}-04)$ and iron ion binding $(\mathrm{P}=4.97 \mathrm{E}-04)$.

The enriched KEGG pathways for downregulated genes are also listed in Table II, including oxidative phosphorylation $(\mathrm{P}=2.49 \mathrm{E}-04)$, Alzheimer's disease $(\mathrm{P}=0.00105)$, Parkinson's disease $(\mathrm{P}=0.00299)$, Huntington's disease $(\mathrm{P}=0.00397)$ and proteasome $(\mathrm{P}=0.008983)$. Furthermore, NADH dehydrogenase ubiquinone flavoprotein 2 (NDUFV2) was revealed to be involved in several neurological diseases, including oxidative phosphorylation, Alzheimer's disease, Parkinson's disease and Huntington's disease.

PPI network analysis. Pyrimidine metabolism, oxidative phosphorylation, Alzheimer's disease and Parkinson's disease were all metabolic pathways associated with neurological diseases. DEGs enriched in these pathways and adrenergic receptor genes $\alpha 1 \mathrm{~d}(A D R A 1 D)$ were used to construct a PPI network. The PPI network consisted of 39 nodes and 183 interactions (Fig. 2). ATP synthase, mitochondrial F1 complex, O subunit (ATP5O; degree, 22) and NDUFV2 (degree, 20) were hub nodes in the PPI network.

With the required confidence threshold of $>0$, the DEGs which had interaction relationships with $A D R A 1 D$ and voltage-dependent L-type calcium channel subunit $\alpha-1 \mathrm{~S}$ (ACNAIS) were indicated in Fig. 3A and B, respectively. DEGs which had interaction relationships with $A D R A 1 D$ and $C A C N A I S$ were merged in Fig. 4. In the PPI network, prohibitin $(P H B)$, oxytocin receptor $(O X T R)$, collapsin response mediator protein $1(C R M P I)$ and dihydropyrimidinase-like 2b (DPYSL2) exhibited an interaction relationship with both $A D R A I D$ and CACNAIS.

\section{Discussion}

The present study indicated that calcium signals in A7r5 cells treated with LPS were weaker when compared with PBS-treated cells (the control group). It has been reported that calcium oxalate crystals are able to trigger inflammation through regulating IL-1 $\beta$ (23). Calcium pyrophosphate has been revealed to induce a novel acute inflammation, pleurisy (23). Thus, LPS induced inflammation in A7r5 cells. In the present study, a total of 2,038 DEGs, including 1,094 upregulated and 944 downregulated genes, were identified in the LPS-treated and control groups. Enrichment analyses indicated that $N D U F V 2$ was involved in several neurological diseases, including oxidative phosphorylation, Alzheimer's disease, Parkinson's disease and Huntington's disease. Furthermore, NDUFV2 (degree, 20) exhibited a higher degree in the PPI network for DEGs enriched in pathways associated with neurological diseases. NDUFV2, which is located on chromosome 18p11.31-p11.2, has been named as a causative gene 


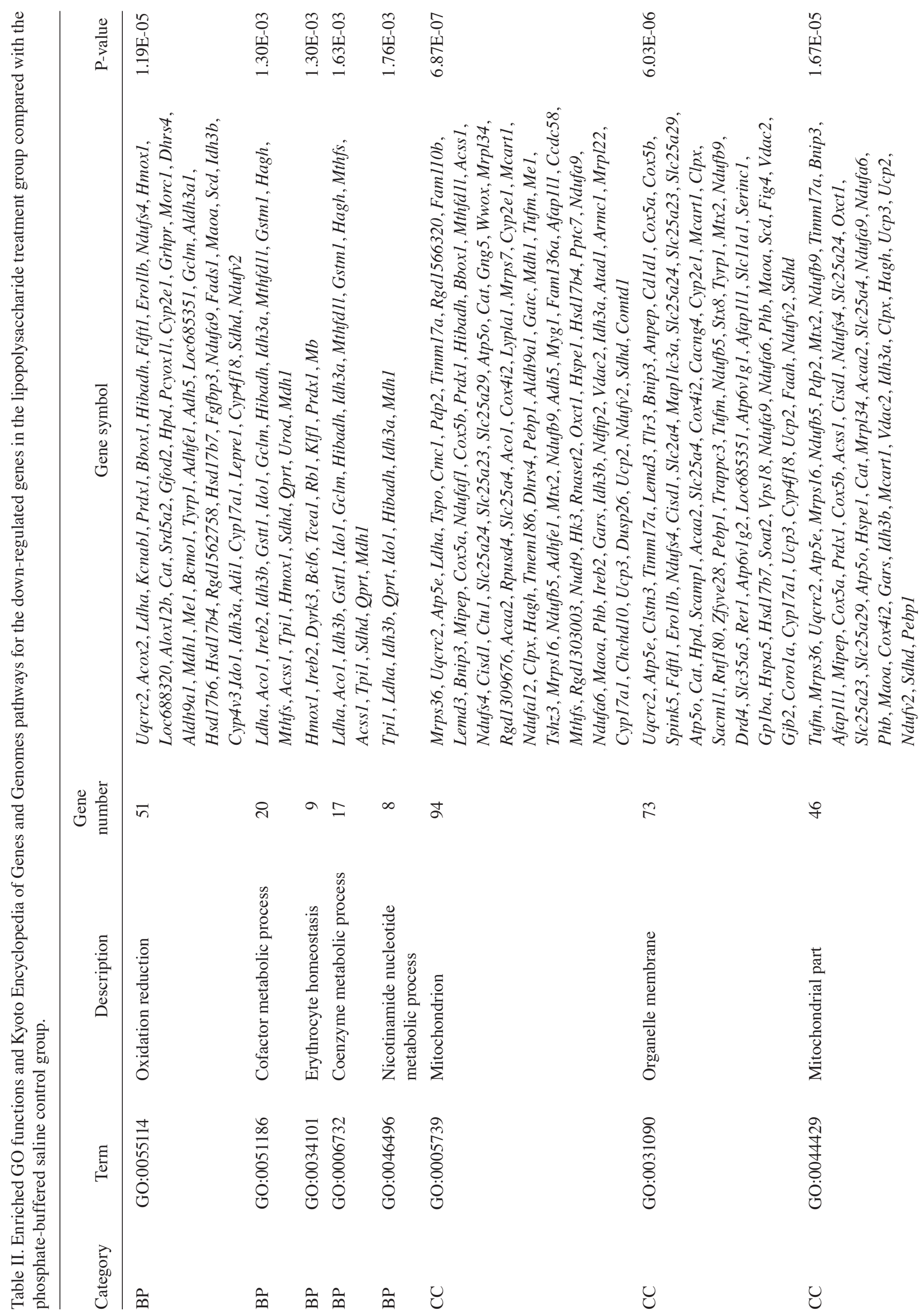




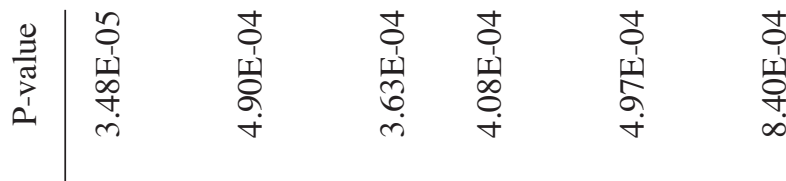

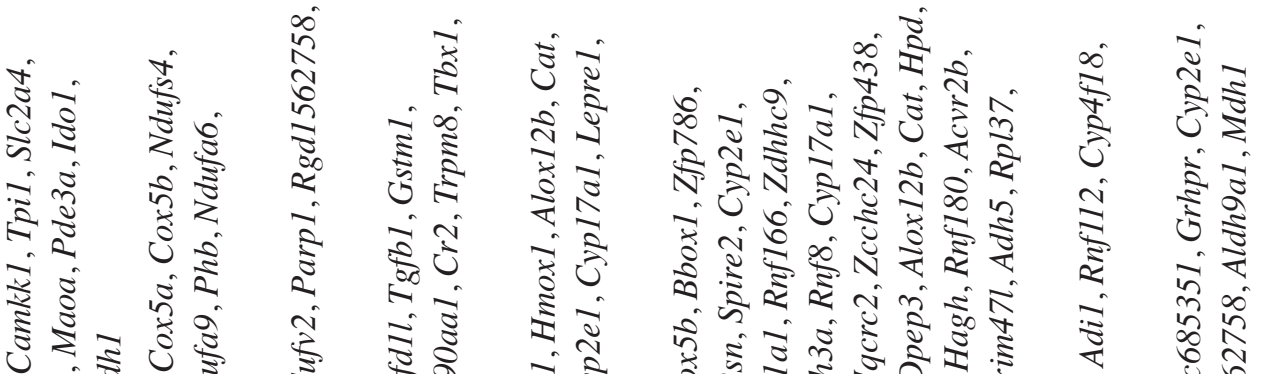

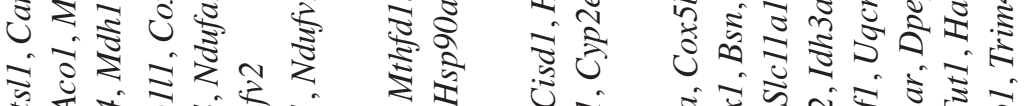

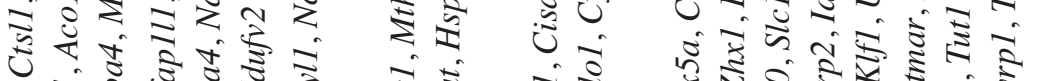

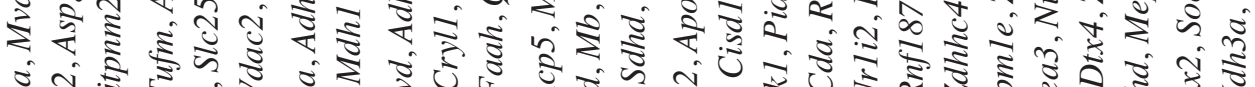

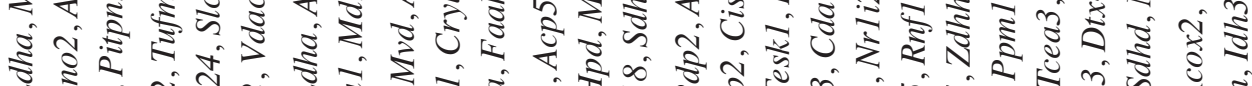

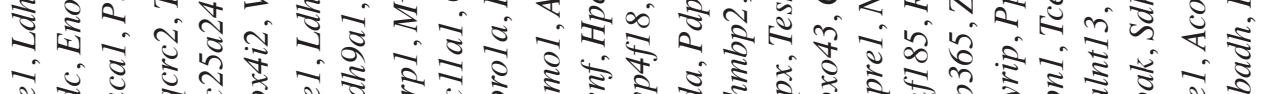

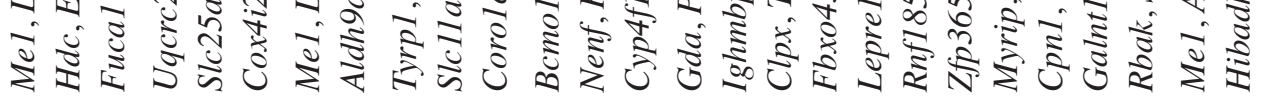

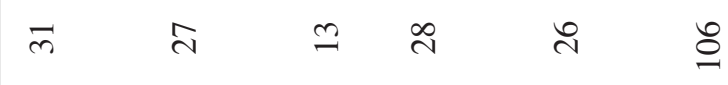



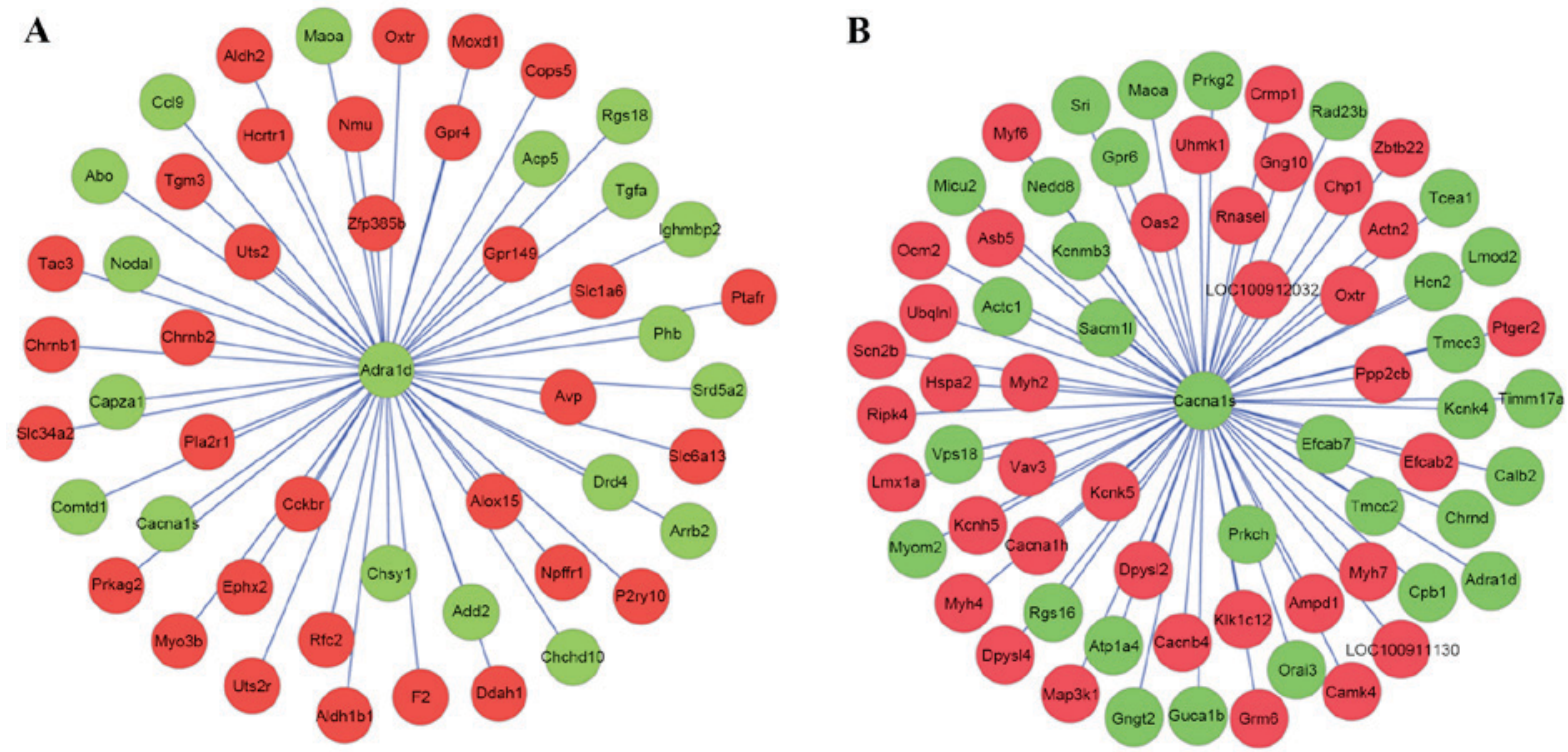

Figure 3. PPI network for ADRA1D/CACNA1S and the DEGs that interact with them. (A) PPI network for ADRA1D and the DEGs that were demonstrated to interact with them. (B) PPI network for CACNA1S and the DEGs interacted with them. Red circles and green circles represent upregulated and downregulated genes, respectively. DEGs, differentially expressed genes; PPI, protein-protein interaction; ADRA1D, adrenoceptor $\alpha$ 1D; CACNA1S, calcium voltage-gated channel subunit $\alpha 1 \mathrm{~S}$.

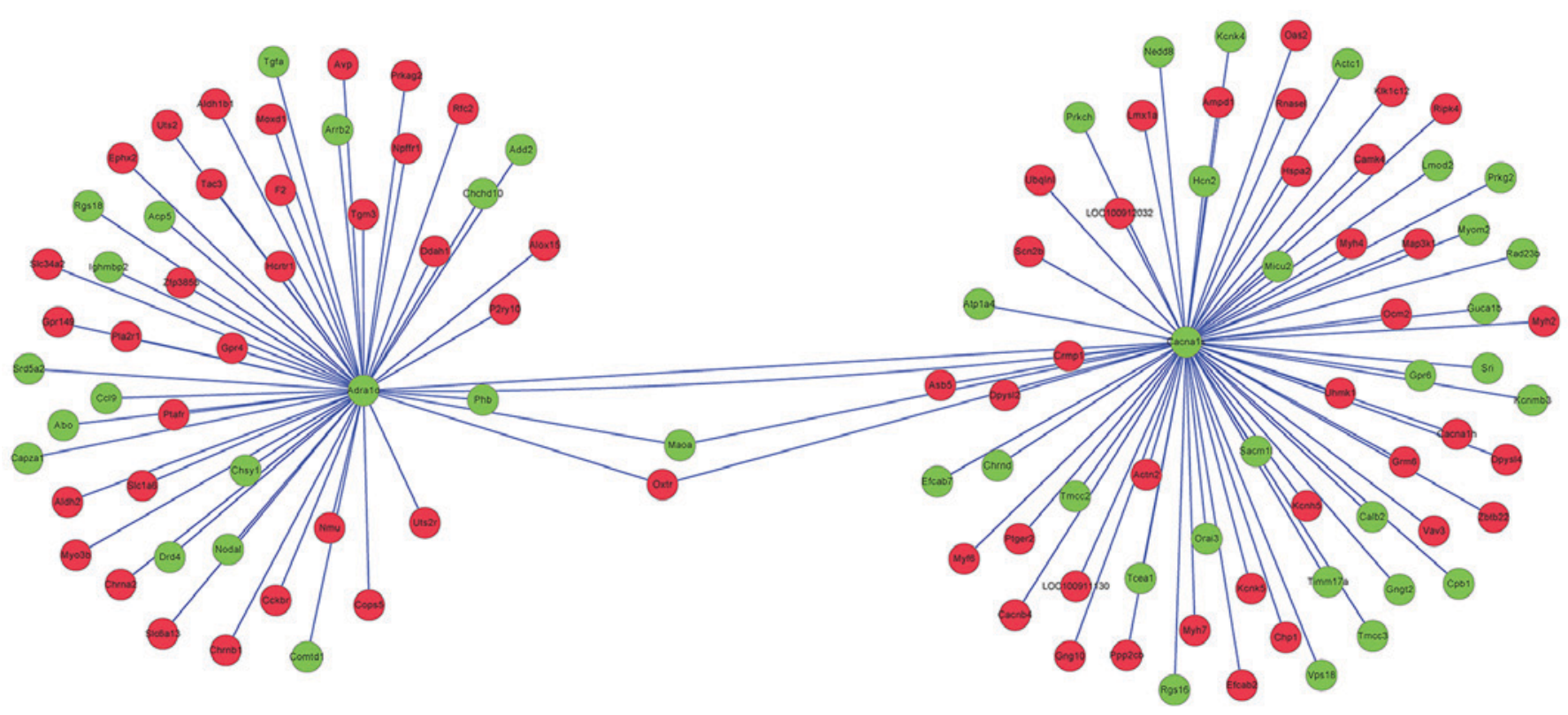

Figure 4. Protein-protein interaction network for ADRA1D/CACNA1S and the differentially expressed genes interact with them. Red circles and green circles represent upregulated and downregulated genes, respectively. ADRA1D, adrenoceptor $\alpha$ 1D; CACNA1S, calcium voltage-gated channel subunit $\alpha 1 \mathrm{~S}$.

in neurological diseases, such as schizophrenia, Parkinson's disease, and bipolar disorder $(24,25)$. A human disease cell model has revealed that the injury of mitochondrial localization of NDUFV2 is related to the pathogenesis of early-onset hypertrophic cardiomyopathy and encephalopathy (26). Therefore, the expression of NDUFV2 may be involved in the effect of inflammation on neurotransmission of VSMC.

In the PPI network for ADRAID, CACNAIS and the DEGs interacting with these components, $P H B, O X T R, C R M P 1$ and $D P Y S L 2$ were revealed to have interaction relationships with both ADRAID and CACNAIS. PHB, a member of the Band-7 family of proteins, has a neuro-damaging role following oxidative and excitotoxic stress, and may serve as target for designing agents to control neuronal death in braininjury, such as cerebral ischemia (27). In cardiomyocytes, overexpressed $P H B$ contributes to the maintenance of the mitochondrial membrane potential and improves cell survival during hypoxia (28). It has been speculated that the function of $P H B$ in protecting against oxidative and hypoxic stress may be correlated with its role in mediating the electron transport chain enzyme, cytochrome C oxidase (29). A previous report has implicated oxytocin $(O X T)$ in inflammatory processes (30). As G-protein coupled receptors, oxytocin receptors (OXTRs) are regulated by G-proteins, which stimulate the phosphatidylinositol-calcium 
secondary messenger system (31). In addition, OXTRs are widely distributed in the central nervous system and mediate various behaviors (32), such as social memory and recognition, responses to stress and anxiety, sexual and maternal behaviors, and bonding (33). These may indicate that the expression levels of $P H B$ and $O X T R$ are correlated with the effect of inflammation on neurotransmission of VSMC.

$C R M P 1$ is affiliated with a cytoplasmic family of proteins and is involved in the development of the central nervous system (34). CRMP1, which may be disturbed by Speedy A1 $(S p y 1)$ from interacting with actin, has a role in the collapse and regeneration of growth cones after sciatic nerve crush (35). Previous studies have identified that within hypertrophic cells of a brain that has suffered a stroke, overexpression of SPNA2 and DPYSL2 (also known as CRMP2) was revealed to be correlated with neurite outgrowth and plasticity, which suggests an early activation of neuronal regeneration, repair and development $(36,37)$. Meanwhile, in the neonatal rat brain, the Akt/glycogen synthase kinase-3 $\beta / C R M P 2$ pathway modulates axonal injury following hypoxia-ischemia $(38,39)$. Thus, the expression levels of CRMP1 and DPYSL2 may be associated with the effect of inflammation on neurotransmission of VSMC.

In conclusion, we screened 2,038 DEGs, including 1,094 upregulated and 944 downregulated genes in the LPS group compared with the control group. The present study identified that NDUFV2, PHB, OXTR, CRMP1 and DPYSL2 may have key roles in the effect of inflammation on the neurotransmission of VSMC. However, the results were speculations following bioinformatics analysis and require further experimental validation.

\section{Acknowledgments}

The present study was supported by Shanghai Science and Technology Commission Fund (grant no. 15495810202) and the Medical Engineering Cross Research Fund, Shanghai Jiaotong University (grant nos. YG2013MS08 and YG2014QN09).

\section{References}

1. Owens GK: Regulation of differentiation of vascular smooth muscle cells. Physiol Rev 75: 487-517, 1995.

2. Fukata Y, Amano M and Kaibuchi K: Rho-Rho-kinase pathway in smooth muscle contraction and cytoskeletal reorganization of non-muscle cells. Trends Pharmacol Sci 22: 32-39, 2001.

3. Arita Y, Kihara S, Ouchi N, Maeda K, Kuriyama H, Okamoto Y, Kumada M, Hotta K, Nishida M, Takahashi M, et al: Adipocyte-derived plasma protein adiponectin acts as a platelet-derived growth factor-BB-binding protein and regulates growth factor-induced common postreceptor signal in vascular smooth muscle cell. Circulation 105: 2893-2898, 2002.

4. Heo SK, Yun HJ, Noh EK, Park WH and Park SD: LPS induces inflammatory responses in human aortic vascular smooth muscle cells via Toll-like receptor 4 expression and nitric oxide production. Immunol Lett 120: 57-64, 2008.

5. Irani K: Oxidant signaling in vascular cell growth, death, and survival a review of the roles of reactive oxygen species in smooth muscle and endothelial cell mitogenic and apoptotic signaling. Circ Res 87: 179-183, 2000.

6. Eltzschig HK and Carmeliet P: Hypoxia and inflammation. N Engl J Med 364: 656-665, 2011.

7. Deten A, Volz HC, Holzl A, Briest W and Zimmer HG: Effect of propranolol on cardiac cytokine expression after myocardial infarction in rats. Mol Cell Biochem 251: 127-137, 2003.
8. Davel AP, Fukuda LE, De Sá LL, Munhoz CD, Scavone C, Sanz-Rosa D, Cachofeiro V, Lahera V and Rossoni LV: Effects of isoproterenol treatment for 7 days on inflammatory mediators in the rat aorta. Am J Physiol Heart Circ Physiol 295: H211-H219, 2008.

9. Zhu Y, Tuerxun A, Hui Y and Abliz P: Effects of propranolol and isoproterenol on infantile hemangioma endothelial cells in vitro. Exp Ther Med 8: 647-651, 2014.

10. Romacho T, Azcutia V, Vázquez-Bella M, Matesanz N, Cercas E, Nevado J, Carraro R, Rodríguez-Mañas L, Sánchez-Ferrer CF and Peiró C: Extracellular PBEF/NAMPT/visfatin activates pro-inflammatory signalling in human vascular smooth muscle cells through nicotinamide phosphoribosyltransferase activity. Diabetologia 52: 2455-2463, 2009.

11. Cuff CA, Kothapalli D, Azonobi I, Chun S, Zhang Y, Belkin R, Yeh C, Secreto A, Assoian RK, Rader DJ and Puré E: The adhesion receptor $\mathrm{CD} 44$ promotes atherosclerosis by mediating inflammatory cell recruitment and vascular cell activation. J Clin Invest 108: 1031, 2001.

12. Dichtl W, Dulak J, Frick M, Alber HF, Schwarzacher SP, Ares MP, Nilsson J, Pachinger O and Weidinger F: HMG-CoA reductase inhibitors regulate inflammatory transcription factors in human endothelial and vascular smooth muscle cells. Arterioscler Thromb Vasc Biol 23: 58-63, 2003.

13. Christodoulou DC, Gorham JM, Herman DS and Seidman J: Construction of normalized RNA-seq libraries for next-generation sequencing using the crab duplex-specific nuclease. Curr Protoc Mol Biol Chapter 4: Unit4.12, 2011.

14. Trapnell C, Pachter L and Salzberg SL: TopHat: Discovering splice junctions with RNA-Seq. Bioinformatics 25: 1105-1111, 2009.

15. D'Antonio M, Picardi E, Castrignanò T, D'Erchia AM and Pesole G: Exploring the RNA editing potential of RNA-Seq data by ExpEdit. Methods Mol Biol 1269: 327-328, 2015.

16. Trapnell C, Roberts A, Goff L, Pertea G, Kim D, Kelley DR, Pimentel H, Salzberg SL, Rinn JL and Pachter L: Differential gene and transcript expression analysis of RNA-seq experiments with TopHat and Cufflinks. Nat Protoc 7: 562-578, 2012.

17. Tarazona S, Furio-Tari P, Ferrer A and Conesa A: NOISeq: Exploratory analysis and differential expression for RNA-seq data. $R$ package version 200, 2012

18. Ashburner M, Ball CA, Blake JA, Botstein D, Butler H, Cherry JM, Davis AP, Dolinski K, Dwight SS, Eppig JT, et al: Gene ontology: Tool for the unification of biology. The Gene Ontology Consortium. Nature genetics 25: 25-29, 2000.

19. Altermann E and Klaenhammer TR: PathwayVoyager: Pathway mapping using the Kyoto Encyclopedia of Genes and Genomes (KEGG) database. BMC Genomics 6: 60, 2005.

20. Huang da W, Sherman BT and Lempicki RA: Systematic and integrative analysis of large gene lists using DAVID bioinformatics resources. Nat Protoc 4: 44-57, 2008.

21. Snel B, Lehmann G, Bork P and Huynen MA: STRING: A web-server to retrieve and display the repeatedly occurring neighbourhood of a gene. Nucleic Acids Res 28: 3442-3444, 2000.

22. Smoot ME, Ono K, Ruscheinski J, Wang PL and Ideker T: Cytoscape 2.8: New features for data integration and network visualization. Bioinformatics 27: 431-432, 2011.

23. Willoughby D, Dunn C, Yamamoto S, Capasso F, Deporter D and Giroud J: Calcium pyrophosphate-induced pleurisy in rats: A new model of acute inflammation. 1975. Agents Actions 43: 221-224, 1994

24. Nishioka K, Vilariño-Güell C, Cobb SA, Kachergus JM, Ross OA, Hentati E, Hentati F and Farrer MJ: Genetic variation of the mitochondrial complex I subunit NDUFV2 and Parkinson's disease. Parkinsonism Relat Disord 16: 686-687, 2010.

25. Washizuka S, Kametani M, Sasaki T, Tochigi M, Umekage T, Kohda K and Kato T: Association of mitochondrial complex I subunit gene NDUFV2 at 18p11 with schizophrenia in the Japanese population. Am J Med Genet B Neuropsychiatr Genet 141B: 301-304, 2006.

26. Liu HY, Liao PC, Chuang KT and Kao MC: Mitochondrial targeting of human NADH dehydrogenase (ubiquinone) flavoprotein 2 (NDUFV2) and its association with early-onset hypertrophic cardiomyopathy and encephalopathy. J Biomed Sci 18: 29, 2011.

27. Teoh J, Boulos S, Chieng J, Knuckey NW and Meloni BP: Over-expressing prohibitin (PHB) in neuronal cultures exacerbates cell death following hydrogen peroxide and L-glutamic acid induced injury. Neurosci Med 5: 149-160, 2014. 
28. Muraguchi T, Kawawa A and Kubota S: Prohibitin protects against hypoxia-induced $\mathrm{H} 9 \mathrm{c} 2$ cardiomyocyte cell death. Biomed Res 31: 113-122, 2010.

29. Tsutsumi T, Matsuda M, Aizaki H, Moriya K, Miyoshi H, Fujie H, Shintani Y, Yotsuyanagi H, Miyamura T, Suzuki T and Koike K: Proteomics analysis of mitochondrial proteins reveals overexpression of a mitochondrial protein chaperon, prohibitin, in cells expressing hepatitis $\mathrm{C}$ virus core protein. Hepatology 50 : 378-386, 2009.

30. Amrani Y, Syed F, Huang C, Li K, Liu V, Jain D, Keslacy S, Sims MW, Baidouri H, Cooper PR, et al: Expression and activation of the oxytocin receptor in airway smooth muscle cells: Regulation by TNFalpha and IL-13. Respir Res 11: 104, 2010.

31. Kimura T, Tanizawa O, Mori K, Brownstein MJ and Okayama H: Structure and expression of a human oxytocin receptor. Nature 356: 356, 1992.

32. Heinrichs M, Baumgartner T, Kirschbaum C and Ehlert U: Social support and oxytocin interact to suppress cortisol and subjective responses to psychosocial stress. Biological Psychiatry 54: 1389-1398, 2003.

33. Thompson RJ, Parker KJ, Hallmayer JF, Waugh CE and Gotlib IH: Oxytocin receptor gene polymorphism (rs2254298) interacts with familial risk for psychopathology to predict symptoms of depression and anxiety in adolescent girls. Psychoneuroendocrinology 36: 144-147, 2011.
34. Li K, Qi Y, Xia T, Yao Y, Zhou L, Lau KM and Ng HK: CRMP1 inhibits proliferation of medulloblastoma and is regulated by HMGA1. PLoS One 10: e0127910, 2015.

35. Yao L, Liu YH, Li X, Ji YH, Yang XJ, Hang XT, Ding ZM, Liu F, Wang YH and Shen AG: CRMP1 interacted with Spy1 during the collapse of growth cones induced by Sema3A and acted on regeneration after sciatic nerve crush. Mol Neurobiol 53: 879-893, 2016.

36. Indraswari F, Wong PT, Yap E, Ng YK and Dheen ST: Upregulation of Dpysl2 and Spna2 gene expression in the rat brain after ischemic stroke. Neurochem Int 55: 235-242, 2009.

37. Tanaka H, Morimura R and Ohshima T: Dpysl2 (CRMP2) and Dpys13 (CRMP4) phosphorylation by Cdk5 and DYRK2 is required for proper positioning of Rohon-Beard neurons and neural crest cells during neurulation in zebrafish. Dev Biol 370: 223-236, 2012.

38. Xiong T, Tang J, Zhao J, Chen H, Zhao F, Li J, Qu Y, Ferriero D and Mu D: Involvement of the Akt/GSK-3 $\beta /$ CRMP-2 pathway in axonal injury after hypoxic-ischemic brain damage in neonatal rat. Neuroscience 216: 123-132, 2012.

39. Zhang CY, Feng SJ, Xu L, Bu XN, Zhang N, Zheng YX, Yuan XW, Li XG and Li JF: CRMP-2 is involved in hypoxic preconditioning-induced neuroprotection against cerebral ischemic injuries of mice. Chin J Basic Clin Med 11: 007, 2009 (In Chinese). 